\title{
Uma proposta de classificação ecográfica mamária
}

\author{
A breast sonography classification proposal
}

\author{
Maria Julia Gregorio Calas ${ }^{1}$, Hilton Augusto Koch², Maria Virginia Peixoto Dutra ${ }^{3}$
}

\section{RESUMO}

Objetivos: as melhorias tecnológicas na qualidade da imagem têm aumentado a importância da ultra-sonografia no estudo das patologias mamárias. A necessidade de padronização para caracterizar, descrever e emitir laudos na análise das imagens motivaram o desenvolvimento de um sistema de classificação de laudos ecográficos mamários. Métodos: o sistema de classificação proposto agrupou as imagens ecográficas mamárias em cinco classes: I - normal, II - benigna, III - indeterminada, IV - suspeita, V - altamente suspeita. As características morfológicas ecográficas utilizadas para a descrição das imagens foram: forma, limites, contorno, ecogenicidade, ecotextura, ecotransmissão, orientação e sinais secundários. O teste padrão, numa casuística de 450 lesões, foi considerado o seguimento ecográfico das lesões por período de 6 a 24 meses e a histopatologia da peça cirúrgica nos casos operados. Resultados: a classificação ecográfica mamária para o diagnóstico de câncer de mama apresentou sensibilidade de 90,2\% (IC: 82,8-94,9\%) e especificidade de 96,2\% (IC: 94,0-97,6\%) O valor preditivo positivo foi de 84,1\% (IC: 76,0-89,9\%) e o valor preditivo negativo foi de 97,8\% (IC: 95,9-98,9\%), alcançando acurácia de 95,1\%. Conclusões: a adoção do sistema de classificação ecográfica resulta na uniformidade e otimização dos laudos. Facilita ainda a comparação com a clínica, com os exames histopatológicos e de imagem mamária, evitando procedimentos desnecessários, conduzindo a condutas terapêuticas mais adequadas.

PALAVRAS-CHAVE: Doenças mamárias/diagnóstico; Ultrasonografia mamária/classificação; Ultrasonografia/classificação

\section{ABSTRACT}

Purpose: the technological improvements in image quality have increased the importance of ultrasound as an imaging method in the study of breast pathologies. The need for a standardized method for lesion characterization, description and reporting in image analysis motivated the development of a breast sonographic report classification system. Methods: the classification grouped the breast sonographic images in five classes: I - normal; II - benign; III - indeterminate, IV - suspect, and V - highly suspect. The used morphologic ultrasound features were shape, border, contour, echogenicity, echotexture, sound transmission, orientation, and secondary signals. The gold standard test, in the study of 450 lesions, considered sonographic follow-up of the lesions for a period from 6 to 24 months and the histopathology of surgical cases. Results: breast sonographic classification for the diagnosis of breast cancer showed a sensitivity of 90.2\% (CI: 82.8-94.9\%), a specificity of $96.2 \%$ (CI: 94.0-97.6\%), a positive predictive value of $84.1 \%$ (CI: $76.0-89.9 \%$ ), and a negative predictive value of $97.8 \%$ (CI: $95.9-98.9 \%$ ), obtaining an accuracy of $95.1 \%$. Conclusions: the adoption of a sonographic classification system results in the standardization and optimization of the reports. It also aids the comparison with clinical findings, histopathological tests and breast images, avoiding unnecessary procedures and therefore leading to more adequate therapeutical management.

KEYWORDS: Breast diseases/diagnosis; Ultrasonography, mammary/classification; Ultrasonography/classification

1 Médica do setor de imaginologia mamária do Centro de Estudos e Pesquisas da Mulher - CEPEM - Rio de Janeiro (RJ) - Brasil.

2 Professor Titular de Radiologia da Faculdade de Medicina da Universidade Federal do Rio de Janeiro - UFRJ - Rio de Janeiro (RJ) - Brasil.

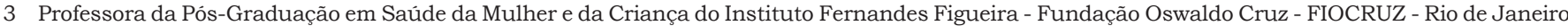
(RJ) - Brasil.

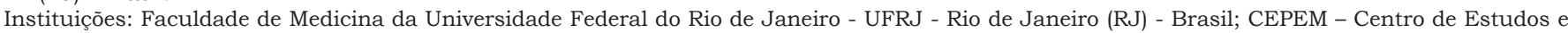
Pesquisas da Mulher - Rio de Janeiro (RJ) - Brasil.

Correspondência: Maria Julia Gregorio Calas

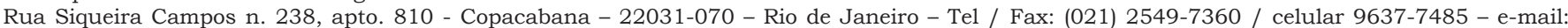
mjgcalas@terra.com.br 
Introdução

O câncer de mama é uma das causas mais importantes de mortalidade entre as mulheres ${ }^{1,2}$, representando o segundo tipo de câncer mais freqüente no mundo e o primeiro entre as mulheres (cerca de 1 milhão de casos novos estimados) ${ }^{1-3}$.

A incidência de câncer de mama feminina apresentou crescimento contínuo na última década, o que pode ser resultado de mudanças sociodemográficas e acessibilidade aos serviços de saúde $^{1,2}$. Seu prognóstico é bom se diagnosticado nos estádios iniciais ${ }^{1,2}$. Estima-se que a sobrevida média geral cumulativa após cinco anos seja de $65 \%$ (variando de 53 a $74 \%$ ) nos países desenvolvidos e de $56 \%$ (variando de 49 a $51 \%$ ) para os países em desenvolvimento ${ }^{3}$. Na população mundial, a sobrevida média após cinco anos é de $61 \%{ }^{3}$. Apesar de ser considerado um câncer de relativamente bom prognóstico, se diagnosticado e tratado oportunamente, as taxas de mortalidade por câncer de mama continuam elevadas no Brasil, muito provavelmente porque a doença ainda seja diagnosticada em estádios avançados ${ }^{3}$.

O número de casos novos de câncer de mama esperados para o Brasil em 2005 é de 49.470, com risco estimado de 53 casos a cada 100 mil mulheres $^{3}$. Na região Sudeste, o câncer de mama é o mais freqüente entre as mulheres, com incidência estimada de 73 casos novos por 100 mil. Sem considerar os tumores de pele não-melanoma, este tipo de câncer também é o mais freqüente nas mulheres das regiões Sul (71/100.000), Centro-Oeste (38/ $100.000)$ e Nordeste $(27 / 100.000)$. Na região Norte é o segundo tumor mais incidente $(15 / 100.000)^{3}$.

Todo esforço para rastrear esta doença na fase inicial é justificável. Na busca do diagnóstico cada vez mais precoce do câncer de mama, a associação de métodos diagnósticos tem sido utilizada com muito êxito ${ }^{2,4}$. No entanto, é importante salientar que o papel de rastreamento do carcinoma mamário é ainda domínio da mamografia ${ }^{1,2}$. Porém, devido fundamentalmente ao desenvolvimento tecnológico observado nessas últimas décadas, a ultra-sonografia mamária tem se tornado método valioso e bem estabelecido no diagnóstico das doenças mamárias ${ }^{4}$. Nos dias de hoje, é inquestionável o seu valor como método complementar à mamografia e à clinica ${ }^{4,5}$. O progresso tecnológico da ultra-sonografia diagnóstica e intervencionista tem grande participação na melhora do prognóstico do câncer de mama ${ }^{1,2,5}$. É exame de execução simples, de realização rápida, sem riscos e, por não utilizar compressão e radiação ionizante, é bem tolerado e aceito pelas pacien- tes $^{1-7}$. Por ser a ultra-sonografia método operadordependende, torna-se patente a necessidade de qualificação e experiência do profissional, que permitirão não apenas a visualização das imagens, mas também a utilização de características ecográficas para inserí-las num sistema de classificação diagnóstica ${ }^{4-7}$.

A interpretação ecográfica tem por base o conhecimento da imagem que representa a estrutura mamária considerada como normal, das variantes do normal e dos aspectos múltiplos da doença da mama. Se uma imagem fora dos padrões de normalidade for identificada, deve ser explorada em todos os planos, com análise do contorno, ecoestrutura e contraste ${ }^{1,4-8}$.

As imagens anormais visualizadas ao estudo ecográfico vêm sendo avaliadas e definidas de acordo com as características morfológicas. O desenvolvimento da capacidade para avaliar os critérios morfológicos das imagens ecográficas e de orientar a diferenciação entre alterações benignas e malignas tem sido objeto de vários estudos. No entanto, existem controvérsias na literatura quanto à capacidade preditiva de cada característica ecográfica para o diagnóstico das doenças mamárias $^{8-12}$. Ainda se fazem necessários novos estudos para aumentar a consistência e a confiabilidade dos sistemas de classificação.

Em face do exposto acima, associado à experiência brasileira na realização de número significativo de exames em uma clínica de imaginologia mamária e à otimização da ultra-sonografia como exame complementar na busca de diagnósticos mais precoces e precisos, os autores deste trabalho se propõem a verificar a validade de nova proposta de classificação ecográfica mamária, comparando com um teste-padrão para o diagnóstico de câncer de mama.

\section{Métodos}

A casuística deste trabalho foi constituída por 637 casos de pacientes encaminhadas por seus médicos assistentes para serem submetidas à punção aspirativa com agulha fina em 206 casos e à biopsia de fragmentos (core biopsia) em 431 casos. Estes procedimentos foram guiados por ultrasonografia e realizados no Centro de Estudos e Pesquisa da Mulher (CEPEM), Rio de Janeiro, no período de janeiro a dezembro de 2003. No exame ultrasonográfico foi utilizado equipamento LOGIC MD $400 \mathrm{GE}$ com transdutor de 7,5 MHz, com registro fotográfico ortogonal de cada imagem avaliada, e material próprio para cada procedimento. 
Dos 637 casos do estudo, 187 foram excluídos da casuística (casos de material insatisfatório de citopunção ou histopatologia). Os 450 casos selecionados foram constituidos por: cistos simples (9 casos), cistos complicados (42 casos), cisto com projeção sólida no interior (1 caso), cisto com septação (1 caso) e lesões sólidas (397 casos).

As características utilizadas na avaliação da morfologia das lesões já foram descritas por vários autore ${ }^{5-12}$ e se encontram discriminadas a seguir: a forma pode ser definida (arredondada, ovóide, alongada) ou indefinida. Os limites podem ser descritos como precisos (nítidos), imprecisos (não nítidos), ou parcialmente precisos. O contorno ou margem pode ser regular, parcialmente regular (podendo apresentar aspecto bocelado ou macrolobulado) ou irregular (podendo apresentar aspecto angular, microlobulado, espiculado ou indistinto). A ecogenicidade é definida pela comparação das imagens com o tecido fibroadiposo circunjacente, conforme descrito por Stavros et al. ${ }^{8}$ e pelo Colégio Americano de Radiologia (ACR) ${ }^{13}$ (BI$\left.\operatorname{RADS}^{\circledR}\right)$, podendo ser anecóica, hipoecóica, isoecóica, hiperecóica e mista (a lesão apresenta dois tipos de ecogenicidade, por exemplo: anecóica e hipoecóica, ou hipoecóica e hiperecóica). A ecotextura é definida como homogênea ou heterogênea. A ecotransmissão pode estar ausente, ou apresentar o reforço acústico posterior ou sombra posterior. A orientação é definida como horizontal ou vertical. Os sinais secundários são representados pelas alterações cutâneas (espessamento da pele maior de $2 \mathrm{~mm}$ e/ou retração), aumento da ecogenicidade do tecido celular subcutâneo, aumento da ecogenicidade peritumoral, aumento difuso da ecogenicidade do parênquima, espessamento dos ligamentos de Cooper, calcificações intratumorais (exceto calcificações grosseiras típicas de fibroadenomas calcificados) e comprometimento do plano muscular e dos linfonodos.

Para melhor entendimento da proposta de classificação aqui utilizada, as imagens ecográficas foram primeiramente analisadas quanto às suas características morfológicas, sendo estas agrupadas e formando um conjunto de critérios ecográficos, sugestivos de benignidade ou malignidade. Partindo do conjunto de critérios ecográficos idealizou-se uma classificação. A capacidade preditiva para malignidade de cada uma das características ecográficas utilizadas nesta classificação foi objeto de outro trabalho.

A proposta de classificação sugerida pelos autores segue os critérios descritos a seguir:

A classe I é constituída por imagens ecográficas que sugerem um padrão de normalidade, com descrição da anatomia ecográfica.
A classe II é constituída por imagens com características de benignidade. As imagens se apresentam com forma bem definida, contorno regular ou bocelado, limites precisos, orientação horizontal, discreto ou acentuado reforço acústico posterior, sombra acústica bilateral ou ausente e ecotextura homogênea, podendo ser anecóicas, hipoecóicas ou isoecóicas. Por vezes, as lesões anecóicas (cistos) podem apresentar ecos móveis no interior, correspondendo a conteúdo espesso ou calcificações (cistos complicados). Algumas imagens hipoecóicas de características benignas podem também apresentar pontos hiperecogênicos no interior, provocando ou não, sombra acústica posterior, correspondendo a nódulo sólido calcificado. Estão ainda incluídos nesta classe os linfonodos intramamários, aglomerado de cistos, ectasia ductal focal ou difusa (quando o eixo ânteroposterior do ducto for maior de $3 \mathrm{~mm}$ ), macrocalcificação isolada (neste caso, é indispensável a comparação com a mamografia), pacientes portadoras de próteses de silicone íntegras ou com imagens sugestivas de alterações da prótese (retração cicatricial da cápsula, herniação do implante, ruptura extracapsular e intracapsular) e imagens sugestivas de seqüelas cirúrgicas em mama operada, associada ou não à radioterapia, como por exemplo, coleções líquidas ou mistas, calcificações, distorção da arquitetura do parênquima e espessamento cutâneo, em correspondência com a cicatriz cirúrgica.

A classe III é constituída por imagens com achados ecográficos de padrão indeterminado. Estão incluídos nesta classe as imagens anecóicas (cistos) com pontos ecogênicos fixos no interior, sugestivas de projeção sólida intracística, ou as imagens anecóicas com espessamento de paredes e septações grosseiras. As imagens nodulares hipoecóicas (sólidas) com as demais características da classe II, porém apresentando apenas uma característica suspeita, ou seja, orientação vertical ou ecotextura heterogênea ou contorno irregular, e as alterações ecográficas dos linfonodos axilares sem lesão intramamária também estão incluídas na classe III. Estas alterações são modificação da forma (adotando uma forma mais redonda), da orientação (adotando uma orientação mais vertical), da ecogenicidade (tornando-se mais hipoecóicos) e alteração ou ausência do hilo.

A classe IV é constituída por imagens com achados ecográficos suspeitos de malignidade. Incluem-se aqui as imagens que possuem duas ou mais características observadas com maior freqüência nas lesões malignas, sendo elas: forma indefinida, contorno irregular, limites parcialmen- 
te precisos ou imprecisos, orientação vertical, ecotextura heterogênea e projeção de sombra acústica irregular ou ausente. A presença de projeção de sombra acústica, sem massa focal e sem história pregressa de trauma ou cirurgia nesse local, também é descrita nesta classe.

A classe $\mathrm{V}$ é constituída por imagens com achados ecográficos altamente suspeitos de malignidade. Estão incluídas nesta classe as imagens descritas na classe IV associadas aos sinais secundários, descritos anteriormente.

A revisão do registro fotográfico, a avaliação da presença dos critérios ecográficos e a aplicação da classificação ecográfica das imagens estudadas (450 imagens) foram realizadas por um dos autores (MJGC). Este autor possui dez anos de experiência em imaginologia mamária e procedimentos invasivos e foi o idealizador do método de classificação.

Este trabalho foi realizado em serviço particular de diagnóstico por imagem, para o qual as pacientes foram encaminhadas por solicitação dos seus médicos e no qual foram considerados para esta pesquisa apenas os exames que apresentaram imagens classificadas conforme a proposta acima. Desse modo não incluímos a classe I, aspecto ecográfico normal. Coube aos médicos assistentes das pacientes utilizar as informações oriundas dos exames (resultados dos procedimentos invasivos) e decidir sobre o tratamento, independente deste estudo, ou seja, coube a eles prosseguir com tratamento cirúrgico ou manter as pacientes em acompanhamento.

Dos 450 casos selecionados com diagnóstico de citopunção e core biopsia satisfatória, o teste-padrão foi composto pelo resultado histopatológico nos casos submetidos à cirurgia (obtidos com os médicos assistentes) e, nos casos não operados, pelo seguimento ecográfico das lesões. Para a determinação de período mínimo adequado de avaliação do seguimento, foi feita média dos períodos adotados pelos diferentes autores ${ }^{7,9-11,14-16}$, que variaram de 6 a 41 meses. Em função destes resultados, foi adotado como tempo de seguimento adequado para esta pesquisa o período de no mínimo 6 meses e no máximo 24 meses. As informações do seguimento foram obtidas nos registros do banco de dados do CEPEM. Nos casos em que as pacientes não retornaram para exame ultrasonográfico no CEPEM, as informações do acompanhamento ecográfico foram fornecidas pelos médicos assistentes.

O projeto foi aprovado sem restrições pelo Comitê de Ética em Pesquisa do Hospital Universitário Clementino Fraga Filho, da Universidade Federal do Rio de Janeiro.
Verificou-se a distribuição de freqüência para cada classe ecográfica proposta, assim como a partir do teste-padrão e calculou-se o percentual de malignidade para cada classe.

Para avaliarmos as propriedades da proposta de classificação foram calculadas a sensibilidade e a especificidade para diagnosticar a malignidade, compreendendo as freqüências das classes II e III como resultado benigno (teste negativo) e das classes IV e V como resultado maligno (teste positivo). Para avaliar a capacidade preditiva (valor preditivo positivo e valor preditivo negativo) utilizou-se a prevalência de câncer presente na população estudada. Foram calculados os intervalos de confiância (IC) utlizando o programa Epi-Info 6.04b CDC-USA, World Health Organization, outubro de 1997. Os resultados de classificação incorreta (falso-positivos e falso-negativos) foram também relatados.

\section{Resultados}

A aplicação do critério de teste padrão numa análise global resultou em $82(18,2 \%)$ casos de câncer e $368(81,8 \%)$ casos de lesões benignas. A distribuição das classes ecográficas segundo os resultados histológicos e seguimento (teste-padrão) está na Tabela 1. Na Tabela 2, apresentamos o número total de casos de câncer, com a respectiva porcentagem, encontrada para cada classe ecográfica nesta casuística.

Tabela 1 - Distribuição das classes ecográficas segundo teste-padrão

\begin{tabular}{|c|c|c|c|c|}
\hline \multicolumn{5}{|c|}{ Teste padrão } \\
\hline \multirow{2}{*}{$\begin{array}{l}\text { Classificação } \\
\text { Ecográfica }\end{array}$} & \multicolumn{2}{|c|}{ Histopatológico } & \multirow[t]{2}{*}{ Seguimento } & \multirow[t]{2}{*}{ Total } \\
\hline & Maligna & Benigna & & \\
\hline Classe II & 02 & $18 \quad 14$ & 307 & 327 \\
\hline Classe III & 06 & 04 & 25 & 35 \\
\hline Classe IV & 60 & 04 & 10 & 74 \\
\hline Classe V & 14 & 00 & 00 & 14 \\
\hline Total & 82 & 26 & 342 & 450 \\
\hline
\end{tabular}

Tabela 2 - Casos de câncer encontrados em cada classe ecográfica

\begin{tabular}{lccc}
\hline $\begin{array}{l}\text { Classificação } \\
\text { ecográfica }\end{array}$ & $\begin{array}{c}\text { Número } \\
\text { de lesões }\end{array}$ & $\begin{array}{c}\text { Número } \\
\text { de câncer }\end{array}$ & $\begin{array}{c}\% \text { de câncer para } \\
\text { cada classe }\end{array}$ \\
\hline Classe II & 327 & 2 & 0,6 \\
Classe III & 35 & 6 & 17,1 \\
Classe IV & 74 & 60 & 81,1 \\
Classe V & 14 & 14 & 100,0 \\
\hline
\end{tabular}


Pode-se verificar que entre as 327 imagens agrupadas como classe II, 307 encontramse em acompanhamento, com mediana de 11 meses, média de 10,5 meses com desvio-padrão de 2 meses. Entre as 20 lesões submetidas à cirurgia, 18 apresentaram laudos de benignidade na peça cirúrgica e duas lesões foram malignas, compativeis com carcinomas papiliferos. Estes dois casos ocorreram em pacientes com idade de 73 e 78 anos, sendo tumores geralmente de bom prognóstico, crescimento lento e que podem apresentar-se de forma definida à ultrasonografia.

Das 35 imagens agrupadas como classe III, 25 lesões foram acompanhadas. Com relação ao seguimento, esta classe apresentou mediana de 12 meses e média de 13 meses, com desvio-padrão de 7 meses. Dez casos prosseguiram para cirurgia, com quatro casos de lesões benignas (um caso de papiloma atípico) e seis casos de lesões malignas, representadas por carcinomas ductais infiltrantes, sendo três casos classificados como carcinomas papiliferos invasivos. As imagens classificadas nesta categoria não apresentam todos os critérios de benignidade, sendo então considerado o termo "padrão indeterminado" apropriado.

Das 74 imagens classificadas como classe IV, 60 casos foram confirmados na peça operatória, quatro foram benignos na cirurgia e 10 não foram submetidas à cirurgia, conduta esta determinada pelo médico asssistente, mantendo-se em acompanhamento sem alteração da imagem com mediana de 24 meses e média de 21 meses com desvio-padrão de 6 meses. Todas as imagens classificadas como classe V, ou seja, lesões com sinais morfológicos suspeitos associados a sinais secundários, foram confirmadas como carcinomas nas peças operatórias.

Para a análise da validade da classificação ecográfica proposta, as classes IV e V foram agrupadas e consideradas classes com achados ecográficos sugestivos de malignidade (teste positivo) e as classes II e III foram agrupadas e consideradas classes com achados ecográficos sugestivos de benignidade (teste negativo). Foram considerados como teste-padrão maligno as lesões com diagnóstico de malignidade na peça operatória, e como teste-padrão benigno as lesões com diagnóstico de peça cirúrgica benigna e seguimento conforme exposto na Tabela 3.
Tabela 3 - Comparação dos resultados da classificação com o teste-padrão

\begin{tabular}{lcccc}
\hline $\begin{array}{l}\text { Classificação } \\
\text { ecográfica }\end{array}$ & $\begin{array}{c}\text { Lesão maligna Lesão benigna Seguimento } \\
\text { Histologia } \\
\text { maligna }\end{array}$ & $\begin{array}{c}\text { Histologia } \\
\text { benigna }\end{array}$ & Total \\
\hline USG positiva & 74 & 4 & 10 & 88 \\
(Classes IV e V) & - & - & - & - \\
USG negativa & 8 & 22 & 332 & 362 \\
(Classes II e III) & - & - & - & - \\
Total & 82 & 26 & 342 & 450 \\
\hline
\end{tabular}

Os resultados obtidos revelaram que, quando os critérios de classificação são aplicados, a sensibilidade foi 90,2\% (IC: $82,8-94,9 \%$ ) e a especificidade 96,2\% (IC: 94,0-97,6\%). Se considerarmos na população estudada uma prevalência de câncer de 18,2\% (82/450), o valor preditivo positivo foi $84,1 \%$ (IC: $76,0-89,9 \%$ ) e o valor preditivo negativo foi $97,8 \%$ (IC: 95,998,9\%), alcançando acurácia de 95,1\%. Encontramos também uma proporção de falso-negativos da classificação de $9,8 \%$ e de falso-positivos de $3,8 \%$.

\section{Discussão}

Em 1995, Stavros et al. ${ }^{8}$ verificaram em 750 lesões sólidas a freqüência da associação de certas características morfológicas com o câncer de mama. Estes autores classificaram as imagens como malignas quando apenas uma característica de malignidade estava presente. Para classificá-las como benignas deveria ser verificada a ausência de qualquer característica de malignidade associada a uma combinação de características de benignidade. Não sendo incluída nos critérios anteriores, a imagem era considerada indeterminada.

Com o melhor conhecimento das imagens e a possibilidade de definirem critérios diagnósticos, diversos autores, em casuísticas diversas, se motivaram a partir do trabalho de Stavros et al. ${ }^{8}$ a propor novas classificações (Quadro 1 ). 
Quadro 1 - Classes ecográficas propostas e probabilidade de câncer de cada classe ecográfica, por autor

\begin{tabular}{|c|c|}
\hline Autor (Ano) & Classes propostas \\
\hline & Benigno $-0,5 \%$ \\
\hline \multirow[t]{4}{*}{ Stavros et al. ${ }^{8}(1995)$} & Indeterminado - 12,3\% \\
\hline & Maligno - 73,0\% \\
\hline & I - Negativo \\
\hline & $\|$ - Benigno $-5 \% \pm 10$ \\
\hline \multirow[t]{4}{*}{ Arger et al. ${ }^{11}$ (2001) } & III - Provavelmente benigno $-10 \% \pm 7$ \\
\hline & IV - Suspeito $-63 \% \pm 26$ \\
\hline & V - Altamente suspeito $-94 \% \pm 4$ \\
\hline & U2 - Provavelmente benigno - $0,7 \%$ \\
\hline \multirow[t]{4}{*}{ Baez et al. ${ }^{17}$ (2005) } & U3 - Indeterminado - 22,8\% \\
\hline & U4 - Suspeito - $84,0 \%$ \\
\hline & U5 - Maligno \\
\hline & I - Normal \\
\hline \multirow[t]{6}{*}{ Pasqualette et al. ${ }^{7}$ (2003) } & II - Benigno - $1 \%$ \\
\hline & III - Indeterminado - 36\% \\
\hline & IV - Suspeito - 96\% \\
\hline & 0 - Incompleto \\
\hline & I - Negativo - 0\% \\
\hline & II - Benigno - 0\% \\
\hline \multirow[t]{6}{*}{ BI-RADS / ACR ${ }^{13}(2003)$} & III - Provavelmente benigno - $\leq 2 \%$ \\
\hline & IV - Suspeito - 3-89\% \\
\hline & V - Altamente suspeito - $\geq 90 \%$ \\
\hline & VI - Malignidade já comprovada \\
\hline & I - Normalll - Benigno - 0\% \\
\hline & III - Provavelmente benigno - 0,4\% \\
\hline \multirow[t]{5}{*}{ Stavros $^{19}(2004)$} & IVa - Suspeito - 10\% \\
\hline & $\mathrm{IVb}$ - Suspeito - $62 \%$ \\
\hline & V-Altamente suspeito - $91 \%$ \\
\hline & II - Benigno - 0,6\% \\
\hline & III - Indeterminado - 17,1\% \\
\hline \multirow[t]{2}{*}{ Calas et al. (presente trabalho) } & IV - Suspeito - $81,1 \%$ \\
\hline & V - Altamente suspeito - $100 \%$ \\
\hline
\end{tabular}

ACR American College of Radiology; BI-RADS - Breast Imaging Reporting and Data System; EUSOMA - European Society of Mastology

A necessidade de objetivar a terminologia diagnóstica nos laudos ecográficos mamários levou Calas et al. ${ }^{6}$ a propor nova classificação. Esta classificação inicial foi composta por quatro categorias: I - normal, II - benigno, III - indeterminado, IV - suspeito, sendo utilizada pela primeira vez, no Rio de Janeiro, em setembro de 1999.

A classificação utilizada pela Sociedade Européia de Mastologia (EUSOMA, 2001), referida por Baez et al. ${ }^{17}$, utiliza as seguintes nomenclaturas: U2 - lesão provavelmente benigna, U3 - indeterminada, U4 - suspeito de malignidade e U5 - acha- dos malignos. Outra classificação, descrita por Michelin e Levy ${ }^{18}$, incluiu a presença de sinais cardinais e secundários, utilizando os seguintes grupos: Grupo E4 - 90\% de malignidade, Grupo E3 - 68,6\%, Grupo E2 - 56,9\% e Grupo E1 - 40,5\% de malignidade. Taylor et al. ${ }^{5}$ utilizaram uma classificação pelo grau de suspeição (LOS - level of suspicion), sendo LOS 1 ou 2 - achados benignos, LOS 4 ou 5 - achados malignos e LOS 3 - quando não pode ser classificada como benigna ou maligna.

No entanto, são muitas as divergências observadas na literatura, e em grande parte são devidas às diferentes metodologias aplicadas em cada estudo, seja nos critérios adotados para selecionar a população ou para classificar as imagens.

A classificação ultra-sonográfica do $\mathrm{ACR}^{13}$ utiliza 6 categorias, semelhantes às categorias da classificação mamográfica. Ainda não foram publicados os resultados da análise da validade desta proposta de classificação, havendo, portanto, oportunidade para seu aperfeiçoamento ${ }^{15,19}$.

Com relação à utilização da categoria 0 em ecografia mamária há controvérsias, principalmente pela ansiedade que resultados como este podem gerar na paciente e, até mesmo, no profissional solicitante. Na proposta de classificação utilizada no nosso trabalho não utilizamos a classe 0 . No estudo ecográfico a imagem é visualizada em tempo real, havendo durante o exame possibilidade de maior ou menor compressibilidade da lesão, rotação do transdutor para melhor avaliação do contorno e estruturas adjacentes à lesão, manipulação do equipamento, uso de recursos como o Doppler, além da possibilidade de palpação da mama da paciente e correlação com cicatrizes cirúrgicas e história clínica. Portanto, ao término do exame ecográfico, o profissional deve estar apto a classificar a imagem descrita, podendo em algumas situações específicas, sugerir outro tipo de exame adequado ao caso.

Stavros ${ }^{19}$ afirmou que raramente utiliza a classe 0 em ultra-sonografia, considerando o estudo ecográfico um método complementar à avaliação mamográfica e exame clinico, sendo necessária a tomada de decisão após o exame, evitando a sugestão de estudos de imagens adicionais.

Para o uso prático de qualquer nomenclatura é importante a definição de termos que ainda causam certa confusão, como por exemplo: complicated cyst e complex cyst ${ }^{20}$, assim como o tipo de imagem que deve ser incluída em cada categoria das classificações propostas. Para Mendelson et al. ${ }^{20}$, cisto complicado é descrito como a presença de ecos internos, homogêneos, associados aos demais critérios de cistos (massa anecóica oval, redonda ou lobulada, contorno circunscrito, com reforço acústico posterior), sendo estas ima- 
gens classificadas como provavelmente benignas. Os cistos complexos são descritos na presença de massa intracística, septações grosseiras, espessamento ou irregularidade do contorno, sendo classificados como suspeitos ${ }^{21}$.

Em revisão dos estudos de Buchberger et al. ${ }^{14}$, Kolb et al. ${ }^{22}$ e Venta et al. ${ }^{23}$, que no total avaliaram 567 cistos complexos, foi identificado somente um caso de carcinoma in situ de $3 \mathrm{~mm}$ (valor preditivo positivo de $0,2 \%$ ).

Em pesquisa realizada por Kemp et al. ${ }^{24}$, de 20 casos de cistos complexos, apenas em dois casos $(0,4 \%)$ confirmou-se malignidade.

Na classificação utilizada neste trabalho, os carcinomas estiveram ausentes em todas as imagens císticas (9 casos) e nos cistos complicados (42 casos), sendo todos classificados como classe II. Dos cistos complexos apenas um caso resultou em carcinoma $(1,9 \%)$. Na comparação destes resultados com os trabalhos publicados, parece aceitável a classificação de cistos simples e cistos complicados na classe II (benigna), sem supervalorizar estes achados e acrescentar ansiedade e custos para a paciente; assim como é possivel a classificação de cistos complexos na classe III (indeterminada). No entanto, estudos posteriores para validação destes dados fazem-se necessários.

No sistema BI-RADS ${ }^{13}$, as lesões sólidas palpáveis são classificadas como categoria 4 ou categoria 5 , indicativas de biopsia. Porém, ainda não foram esclarecidos quais critérios estariam relacionados a um risco maior para malignidade (tamanho da lesão relativamente ao tamanho da mama). Acredita-se que, com o uso da proposta de classificação do sistema BI-RADS ${ }^{13}$, número maior de pacientes seriam submetidas a acompanhamento em curto prazo, ou seja, estariam excluídos do seguimento apenas os cistos simples, assim como número maior de pacientes seriam submetidas a procedimentos invasivos ou cirúrgicos.

Stavros ${ }^{19}$, em função da experiência adquirida, sugere algumas adaptações à proposta de classificação do sistema BI-RADS ${ }^{13}$. Este autor dividiu a categoria 4 em dois subgrupos: $4 a$ - risco de malignidade maior que $2 \%$ e menor que $50 \%$ e $4 \mathrm{~b}-$ risco de 50 a $90 \%$. Esta divisão é também subjetiva, visto que não há descrição das imagens correspondentes a cada uma destas classes ecográficas.

No nosso trabalho optou-se por não subdividir a classe IV, porque a conduta nesta classe é prosseguir com a investigação, evitando divergências e maiores confusões para os médicos que recebem estes laudos.

Por conseguinte, para futuras avaliações sugerimos que seja considerada a seguinte divisão: classe I normal, classe II provavelmente benigno, classe III padrão indeterminado, classe IV suspei- ta, sem subdivisões, pois uma lesão não pode ser definida como sendo "muito ou pouco" suspeita, e finalmente a classe V como altamente suspeita.

$\mathrm{Na}$ Tabela 4 estão expostas as classificações propostas por cada autor e as probabilidades de câncer calculadas para cada classificação.

Apenas a classificação ecográfica do BIRADS $^{13}$ apresenta $0 \%$ de câncer para a categoria I, no entanto ainda não há dados publicados que confirmen estes resultados. Os demais autores não apresentam esta probabilidade pelo fato de não incluírem a categoria I, ou seja, estudo ecográfico normal, nas casuísticas publicadas.

Com relação à classe II, Pasqualette et al. ${ }^{7}$ encontraram dois casos (1\%) de carcinomas, um caso de carcinoma mucinoso e um caso de carcinoma medular. Estes tipos histológicos de câncer, de forma semelhante ao que ocorre na mamografia, podem apresentar critérios de benignidade. Estes achados estão em correspondência com o trabalho de avaliação interobservador realizado por Rahbar et al. ${ }^{12}$, no qual em um caso de carcinoma medular, os três observadores classificaram a imagem como benigna, assim como no trabalho de Paulinelli et al. ${ }^{15}$, em que foram encontrados três casos $(2,3 \%)$ de tumores malignos entre 133 imagens que não apresentaram nenhuma característica de malignidade. Embora nos estudos que envolvem interpretação e classificação de imagens ultra-sonográficas seja recomendada a análise da concordância entre observadores e a generalização baseada em estudos com apenas um observador seja limitada, Buchberger et al. ${ }^{14}$, Skaane e Engedal ${ }^{25}$, Zonderland et al. ${ }^{9}$ e Chen et al. ${ }^{21}$ consideraram que estudos realizados por apenas um observador podem ser consistentes na aplicação de critérios para a definição de uma lesão e na análise da validade de uma classificação.

A classe II deste trabalho apresenta probabilidade de câncer $(0,6 \%)$ semelhante à proposta inicial de Stavros et al. ${ }^{8}(0,5 \%)$ e à proposta de classificação da Sociedade Européia de Mastologia ${ }^{17}(0,7 \%)$.

Com relação à análise da classe III, existe variabilidade na literatura. Nos estudos de Stavros e cols. ${ }^{8,19}$ houve diferenças nas publicações, apresentando a primeira ${ }^{8}$ a probabilidade de $12,3 \%$ de câncer para o padrão indeterminado e a segun$\mathrm{da}^{19}$ de $0,4 \%$ para a categoria III. No trabalho de Pasqualette et al. ${ }^{7}$ encontramos a probabilidade de $36 \%$ de câncer para a classe III. Na proposta atual de classificação esta porcentagem diminuiu para $17,1 \%$, em correspondência com os achados de Arger et al. ${ }^{11}(10 \% \pm 7)$. Isto pode ter ocorrido em função da experiência adquirida durante o período decorrido após as primeiras publicações. Aparentemente a classificação atual discrimina melhor o câncer, mas é recomendável estabelecer 
comparações estatísticas levando em conta a prevalência global de câncer nas casuísticas.

Com relação às classes IV e $\mathrm{V}$, encontramse resultados variando entre 63 e $100 \%$, com exceção da categoria 4 do BI-RADS ${ }^{13}$, no qual a probabilidade de câncer pode variar de 3 a 90\%, uma faixa muito ampla, fato que demanda melhor definição desta categoria.

O Quadro 2 exemplifica os trabalhos nos quais houve a oportunidade de serem avaliadas as propriedades diagnósticas da ultra-sonografia na detecção de câncer. Deve-se ficar atento à prevalência de câncer encontrada em cada estudo, principalmente para efetuar a comparação dos valores preditivos ${ }^{6,8-12,15}$.

Quadro 2 - Propriedades diagnósticas da ultra-sonografia mamária, por autor.

\begin{tabular}{|c|c|c|c|c|c|c|}
\hline Autor (Ano) & Sens & Esp & Prev & VPP & VPN & Ac \\
\hline & $\%$ & $\%$ & $\%$ & $\%$ & $\%$ & $\%$ \\
\hline Stavros et al. ${ }^{8}$ (1995) & 98,4 & 67,8 & 16,7 & 38,0 & 99,5 & 72,9 \\
\hline Yang et al. ${ }^{16}(1996)$ & 97,0 & 96,8 & 16,4 & 85,3 & 99,4 & 97,0 \\
\hline Chao et al. ${ }^{26}(1999)$ & 86,1 & 66,1 & 23,7 & 44,1 & 93,9 & 70,8 \\
\hline Skaane et al..$^{25}$ (1998) & 99,5 & 29,0 & 57,7 & 66,0 & 98,0 & - \\
\hline Buchberger et al..$^{14}$ (1999) & 92,9 & 76,3 & 6,5 & 25,3 & 99,2 & 77,6 \\
\hline Baez et al. ${ }^{17}(2005)$ & 96,7 & 81,7 & 15,0 & - & 99,3 & - \\
\hline Pasqualette et al. ${ }^{7}$ (2003) & 97,3 & 86,1 & 26,5 & 71,7 & 98,9 & 89,1 \\
\hline Stavros $^{19}(2004)$ & 99,8 & 30,5 & 32,8 & 42,1 & 99,6 & 53,8 \\
\hline Chen et al. ${ }^{21}$ (2004) & 79,3 & 89,3 & 32,5 & 78,1 & 90,0 & 86,0 \\
\hline Paulinelli et al. ${ }^{15}$ (2005) & 96,1 & 60,2 & 26 & 45,9 & 97,7 & 69,8 \\
\hline Calas et al. (presente trabalho) & 90,2 & 96,2 & 18,2 & 84,1 & 97,8 & 95,1 \\
\hline
\end{tabular}

Sens = sensibilidade; Esp $=$ especificidade; Prev $=$ prevalência; VPP $=$ valor preditivo positivo, VPN = valor preditivo negativo; $A c=$ acurácia.

A sensibilidade de um teste expressa a probabilidade de este teste ser positivo dada a presença da doença. Os testes com maior sensibilidade são utilizados para rastreamento de uma doença em grupos populacionais, como por exemplo, a mamografia. Já a especificidade expressa a probabilidade de um teste ser negativo dada a ausência da doença. Os testes com maior especificidade são utilizados para confirmar um diagnóstico e orientar uma conduta, como por exemplo, uma punção percutânea ou um procedimento cirúrgico. No Quadro 2 pode-se observar certa homogeneidade entre os valores de sensibilidade, de forma que os valores de sensibilidade foram mais altos que os valores de especificidade, exceto no trabalho de Chen et al. ${ }^{21} \mathrm{e}$ no nosso trabalho, em que a especificidade $(96,2 \%)$ foi maior que a sensibilidade $(90,2 \%)$.

O valor preditivo positivo é a proporção de verdadeiros positivos entre todos os indivíduos com teste positivo, sendo que o nosso trabalho e o de Yang et al. ${ }^{16}$ apresentam os melhores resultados (Quadro 2). Os valores preditivos positivos dos ou- tros estudos são em geral baixos, alcançando no máximo $78,1 \%$ de probabilidade de câncer diante de uma classificação ecográfica positiva, mesmo sabendo-se que maiores prevalências tendem a aumentar os valores preditivos positivos. O valor preditivo negativo é a proporção de verdadeiros negativos entre todos os indivíduos com teste negativo. Tal como as sensibilidades, os valores preditivos negativos do Quadro 2 se revelaram também muito homogêneos, variando de 90,0 a 99,5\%. Finalmente, a acurácia, que é definida como a proporção de acertos de um teste diagnóstico, teve os maiores valores nos trabalhos de Yang et al. ${ }^{16}$ $(97,0 \%)$ e no nosso trabalho atual $(95,1 \%)$.

A utilização de uma padronização na descrição das lesões mamárias e de uma classificação ecográfica é importante, pois possibilita uma análise das imagens de maneira mais objetiva e cria uma nomenclatura uniforme. Além disso, possibilita a emissão de laudos conclusivos, com uma impressão diagnóstica, capaz de indicar o grau de suspeição para malignidade de acordo com a morfologia dos sinais ecográficos encontrados. Por conseguinte permite orientar de forma mais precisa a conduta a ser seguida, beneficiando desta maneira todas as mulheres. Possibilita ainda a correlação com imagens mamográficas, exame clínico e resultados citológicos e histopatológicos. Resulta, também, em acentuada economia de tempo por parte de quem interpreta tais exames e maior entendimento por parte de quem os solicita. É um meio importante para avaliar a incidência de resultados falso-negativos e falso-positivos e facilita o manuseio dos resultados quando se necessita realizar estudos retrospectivos do material arquivado. O uso de uma classificação pressupõe um compromisso com o diagnóstico.

Na medida em que a proposta de classificação utilizada neste trabalho seja objeto de um estudo multiinstitucional, não apenas um maior número de casos, mas sua utilização ampliada, permitiria uma análise mais definitiva, com dados para uma reavaliação e, conseqüentemente, confirmação da validade e confiabilidade do sistema de classificação ecográfica, assim como forneceria as evidências necessárias para a recomendação de conduta para cada classe.

\section{Agradecimentos}

Ao Dr. Henrique Alberto Pasqualette, diretor do CEPEM, Dr. Paulo Mauricio Soares-Pereira, pela revisão deste trabalho, às Dras. Rosana Castro, Valeska Caldoncelli, Vânia Ravizzini, Madelon Fernandes e Laila Feld, pela realização dos exames ecográficos, $e$ a toda equipe de enfermagem do CEPEM. 


\section{Referências}

1. Paulinelli RR, Moreira MAR, Freitas-Júnior R. Ultra-sonografia no diagnóstico do câncer de mama: realidade atual e possibilidades para o futuro. Rev Bras Mastol. 2003;13(4):168-74.

2. Paulinelli RR, Moreira MAR, Freitas-Júnior R. A importância do diagnóstico precoce do câncer de mama. Femina. 2004;32(3):233-7.

3. Ministério da Saúde. Instituto Nacional de Câncer (INCA) [sitio na Internet]. Estimativa 2005: incidência de câncer no Brasil. Rio de Janeiro: Ministério da Saúde/Secretaria de Vigilância em Saúde/Secretaria de Atenção à Saúde/Instituto Nacional de Câncer/Coordenação de Prevenção e Vigilância; 2004 [citado 2005 Jan 3]. Disponivel em: http:/ / www.inca.gov.br

4. Fonseca ALA. Ultra-sonografia da mama. In: Pasqualette HA, Koch HA, Soares-Pereira PM, Kemp C, editores. Mamografia atual. Rio de Janeiro: Revinter; 1998. p. 205-15.

5. Taylor KJ, Merritt C, Piccoli C, Schmidt R, Rouse $\mathrm{G}$, Fornage B, et al. Ultrasound as a complement to mammography and breast examination to characterize breast masses. Ultrasound Med Biol. 2002;28(1):19-26.

6. Calas MJG, Castro R, Manoel VR, Pasqualette HA, Soares-Pereira PM. Proposta de normatização dos laudos de ultra-sonografia mamária. Femina. 2002;30(2):103-10.

7. Pasqualette HAP, Soares-Pereira PM, Calas MJG, Santos RCR, Manoel VR, Caldoncelli V, et al. Revisão e validação de uma proposta de classificação de laudos de ultra-sonografia mamária. Rev Bras Mastol. 2003;13(4):159-67.

8. Stavros AT, Thickman D, Rapp CL, Dennis MA, Parker SH, Sisney GA. Solid breast nodules: use of sonography to distinguish between benign and malignant lesions. Radiology. 1995;196(1):123-34.

9. Zonderland HM, Hermans J, Coerkamp EG. Ultrasound variables and their prognostic value in a population of 1103 patients with 272 breast cancers. Eur Radiol. 2000;10(10):1562-8.

10. Paulinelli RR, Vidal CSR, Ruiz AN, Moraes VA, Bernardes-Júnior JRM, Freitas-Júnior R. Estudo prospectivo das características sonográficas no diagnóstico de nódulos sólidos da mama. Rev Bras Ginecol Obstet. 2002;24(3):195-9.

11. Arger PH, Sehgal CM, Conant EF, Zuckerman J, Rowling SE, Patton JA, et al. Interreader variability and predictive value of US descriptions of solid breast masses: pilot study. Acad Radiol. 2001;8(4):335-42.

12. Rahbar G, Sie AC, Hansen GC, Prince JS, Melany $\mathrm{ML}$, Reynolds HE, et al. Benign versus malignant solid breast masses: US differentiation. Radiology. 1999;213(3):889-94.
13. American College of Radiology. Illustrated Breast Imaging Reporting and Data System Atlas (BIRADS $\left.^{\circledR}\right)$ : ultrasound. 4th ed. Reston: American College of Radiology (ACR); 2003.

14. Buchberger W, Dekoekkoek-Doll P, Springer P, Obrist $\mathrm{P}$, Dünser $\mathrm{M}$. Incidental findings on sonography of the breast: clinical significance and diagnostic workup. AJR Am J Roentgenol. 1999;173(4):921-7.

15. Paulinelli RR, Freitas-Júnior R, Moreira MA, Moraes VA, Bernardes-Júnior JR, Vidal CS, et al. Risk of malignancy in solid breast nodules according to their sonographic features. J Ultrasound Med. 2005;24(5):635-41.

16. Yang WT, Mok CO, King W, Tang A, Metreweli C. Role of high frequency ultrasonography in the evaluation of palpable breast masses in Chinese women: alternative to mammography? J Ultrasound Med. 1996;15(9):637-44.

17. Baez E, Strathmann K, Vetter M, Madjar H, Hackelöer BJ. Likelihood of malignancy in breast lesions characterised by ultrasound with a combined diagnostic score. Ultrasound Med Biol. 2005;31(2):179-84.

18. Michelin J, Levy L. Ultra-sonografia da mama diagnóstica e intervencionista. Rio de Janeiro: MEDSI; 2001.

19. Stavros AT. Ultrasound of solid breast nodules: distinguishing benign from malignant. In: Stavros AT, editor. Breast ultrasound. Philadelphia: Lippincott Williams \& Wilkins; 2004. p. 445-527.

20. Mendelson EB, Berg WA, Merritt CR. Toward a standardized breast ultrasound lexicon, BI-RADS: ultrasound. Semin Roentgenol. 2001;36(3):217-25.

21. Chen SC, Cheung YC, Su CH, Chen MF, Hwang TL, Hsueh S. Analysis of sonographic features for the differentiation of benign and malignant breast tumors of different sizes. Ultrasound Obstet Gynecol. 2004;23(2):188-93.

22. Kolb TM, Lichy J, Newhouse JH. Occult cancer in women with dense breasts: detection with screening US: diagnostic yield and tumor characteristics. Radiology. 1998;207(1):191-9.

23. Venta LA, Kim JP, Pelloski CE, Morrow M. Management of complex breast cysts. AJR Am J Roentgenol. 1999;173(5):1331-6.

24. Kemp C, Elias S, Borelli K, Garcia y Narvaiza D, Kemp CGM, Schor AP. Punção aspirativa por agulha fina orientada por ultra-sonografia em lesões não-palpáveis. Rev Bras Ginecol Obstet. 2001;23(5):321-7.

25. Skaane P, Engedal K. Analysis of sonographic features in the differentiation of fibroadenoma and invasive ductal carcinoma. Am J Roentgenol. 1998;170(1):109-14.

26. Chao TC, Lo YF, Chen SC, Chen MF. Prospective sonographic study of 3093 breast tumors. J Ultrasound Med. 1999;18(5):363-70; quiz 371-2. 